\title{
A ROTATABLE ELECTRON SPECTROMETER SYSTEM FOR SPIN- AND ANGLE-RESOLVED PHOTOEMISSION EXPERIMENTS WITH CIRCULARLY POLARIZED SYNCHROTRON RADIATION
}

\author{
Ch. HECKENKAMP ${ }^{1)}$. A. EYERS ${ }^{1)}$, F. SCHÄFERS ${ }^{2)}$, G. SCHÖHENSE ${ }^{1)}$ and U. HEINZMANN ${ }^{1)}$ \\ I) Fritz-Haber-Institut der MPG, Faradayweg 4-6, D-1000 Berlin 33, Germany and \\ Fakultät für Physik der Universität Bielefeld, D-4800 Bielefeld 1, FRG \\ 2) BESSY, Lentzeallee 100, D-1000 Berlin 33, Germany.
}

An electron-optical system is described which has been especially designed for spin- and angle-resolved photoelectron spectroscopy with circularly polarized synchrotron radiation. One hv- and one uhv-type of this system (for studies at atoms/molecules and solids/adsorbates, respectively) are in operation at the storage ring BESSY. The system employs, thus, a light source and an electron spin detector (high energy Mott detector) both fixed in space. This is realized by a rotatable electron spectrometer and a $90^{\circ}$ deflector by which the electron beam is directed along the axis of rotation of the spectrometer, independent of the emission angle chosen.

It is the purpose of this paper to describe an electron-optical system which has been designed for spinand angle-resolved photoelectron spectroscopy with circularly polarized synchrotron radiation. The system can be used, however, whenever the capability of angle resolution is required and the light source as well as the electron detector must be kept fixed.

Fig. 1 shows a schematic diagram of the device. The photon source used is the $6.5 \mathrm{~m}$ normal-incidence monochromator [1] at the storage ring BESSY in Berlin, which utilizes a unique property of synchrotron radiation, namely the high degree of circular polarization of the radiation emitted above and below the storage ring plane. With present running conditions $(600 \mathrm{~mA}$ beam current), monochromatized synchrotron radiation with a bandwidth of $\Delta \lambda=0.2 \mathrm{~nm}$, circular polarization of $92 \%$ and a photon flux of more than $5 \times 10^{11}$ photons $/ \mathrm{s}$ in the wavelength range from 45 to $200 \mathrm{~nm}$ is available behind the exit slit. The light beam hits the phototarget and produces photoelectrons in a region shielded against electric and magnetic fields. The spin orientation of the photoelectrons is described by the spin polarization vector $\boldsymbol{P}$, which can be decomposed into the components $A(\theta)$ parallel to the photon momentum, $P_{\perp}(\theta)$ perpendicular to the reaction plane (which is spanned by the momentum of the incoming photon and the outgoing photoelectron) and $P_{\mathrm{p}}(\theta)$ in the reaction plane but perpendicular to the photon momentum (not shown in fig. 1). The formulae for the angle dependences of these components (given by Huang [2]) are relatively complex for the general case if elliptically polarized radiation is used. They become, however, much simpler for the case when the angle between the reaction plane and the major axis of the light ellipse is $45^{\circ}$, as shown in fig. 1. In particular, the differential cross section, which appears as the denominator in the spin polarization formulae, contains neither the degree of polarization of the radiation nor the azimuthal orientation of the reaction plane:

$$
\frac{\mathrm{d} \sigma}{\mathrm{d} \Omega}(\theta)=\frac{Q}{4 \pi}\left(1-\frac{\beta}{2} P_{2}(\cos \theta)\right)=\frac{Q}{4 \pi} F(\theta, \beta),
$$

where $Q$ is the partial cross section, $P_{2}(\cos \theta)$ the second Legendre polynomial and $\beta$ the asymmetry

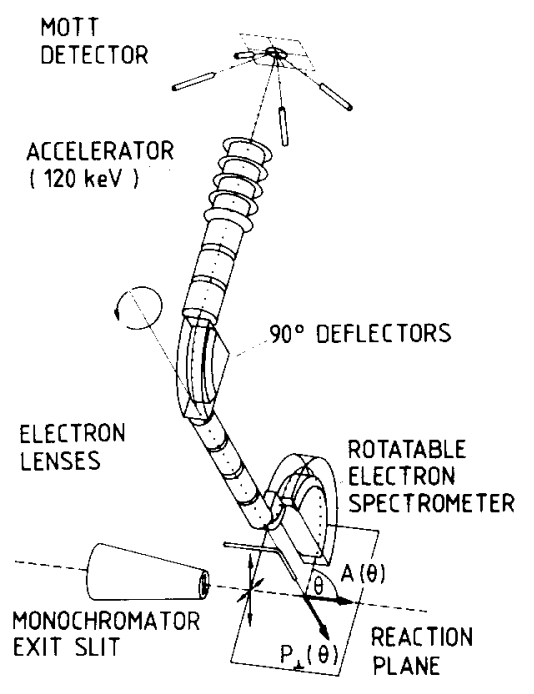

Fig. 1. Schematic diagram of the apparatus. 
parameter of the differential cross section.

The photoelectron spin polarization components are characterized by the dynamical spin parameters $A, \alpha$ and $\xi$, which can be obtained from angle-resolved measurements of the two components shown in fig. 1:

$P_{\perp}(\theta)=\frac{2 \xi \sin \theta \cos \theta}{F(\theta, \beta)}$,

$A(\theta)=\gamma\left(P_{\text {circ }}\right) \frac{A-\alpha P_{2}(\cos \theta)}{F(\theta, \beta)} P_{\operatorname{lin}} \frac{2 \xi \sin ^{2} \theta}{F(\theta, \beta)}$,

where $P_{\text {circ }}$ and $P_{\text {lin }}$ are the degrees of circular and linear light polarization (which are routinely measured by means of a four-mirror analyzer), respectively, and $\gamma$ is the helicity of the radiation $\left(\gamma=+1\right.$ for $\sigma^{+}$light, i.e. photon spin and photon momentum are parallel, $\gamma=-1$ for $\sigma^{-}$light). The component $P_{\mathrm{p}}(\theta)$ contains no further dynamical spin parameters.

The spin parameters $A, \alpha$ and $\xi$ can be extracted from measurements of $P_{\perp}(\theta)$ and $A(\theta)$. Without going fully into details of the experimental procedure, we note that the second term in eq. (3) does not depend upon the helicity of the ionizing radiation and can thus be eliminated by measuring $A(\theta)$ using $\sigma^{+}$and $\sigma^{-}$light. When in addition the emission angle $\theta_{\mathrm{m}}=54.7^{\circ}$ is chosen, where $P_{2}\left(\cos \theta_{\mathrm{m}}\right)=0$, the spin polarization values $P_{\perp}\left(\theta_{\mathrm{m}}\right)$ and $A\left(\theta_{\mathrm{m}}\right)$ are simply proportional to the spin parameters $\xi$ and $A$, respectively. Only the determination of $\alpha$ requires measurements at other angles than the "magic angle" $\theta_{\mathrm{m}}$. Finally, it is worth noting that $\xi$ can be determined with unpolarized light, whereas for the determination of $A$ and $\alpha$ the use of elliptically polarized light is absolutely essential.

Besides the requirement for simple equations, allowing a simple data analysis and saving valuable acquisition time, mechanical restrictions were of importance in designing the electron spectrometer system: both the light source and the spin polarization detector are fixed in space and cannot be rotated to probe different emission angles $\theta$ of the photoelectrons. The first restriction is clear from the fact that synchrotron radiation from a storage ring is used. The second originates from the decision to use high-energy Mott scattering at $120 \mathrm{keV}$ electron energy for spin polarization analysis [3]. A high-energy Mott detector requires an accelerator for high voltage and is an instrument of a volume of about $1 \mathrm{~m}^{3}$. It does not need uhv conditions, however, and, once calibrated, gives absolute spin polarization values with excellent long-term stability, which are important advantages especially for gas-phase photoionization experiments.

The problem of selecting different emission angles, although light source and detector are fixed, was solved by using a rotatable, deflection-type electron spectrometer in combination with a second deflection, which forces the electron beam to coincide with the rotational axis of the system: the photoelectrons emerging under an angle $\theta$ in the reaction plane are energy analyzed by means of a rotatable hemispherical electron spectrometer. An electrostatic deflection by $90^{\circ}$ directs the electron beam along the axis of rotation of the electron spectrometer. After a second deflection by $90^{\circ}$ the electron beam is accelerated to $120 \mathrm{keV}$ and scattered at the gold foil of the Mott detector, where the spin polarization components $A(\theta)$ and $P_{\perp}(\theta)$, then being transverse, are simultaneously determined from the left-right scattering asymmetry measured by two pairs of counters [4].

Fig. 2 shows the gas-phase version of the instrument built. A second system with minor geometrical changes has been built on uhv standards for application in photoemission from solids and adsorbates. Fig. 3 shows the entrance stage of the uhv version with the baffle system for the case of normal light incidence and normal electron emission: it allows the light beam to pass the spectrometer without producing background electrons.

From the number of known spectrometer types, the simulated hemispherical electron analyzer [5] has been chosen, which produces a point image of a point source and thereby simplifies the further transport of the monochromatized electron beam. Key parameters of the spectrometer are given in table 1 . Special attention was paid to design a system which allows the detection of electrons with very low kinetic energies (below $1 \mathrm{eV}$ ). From this point of view, the hemispherical analyzer, where the electrons travel along an equipotential surface, seems to be preferred above a mirror-type analyzer,

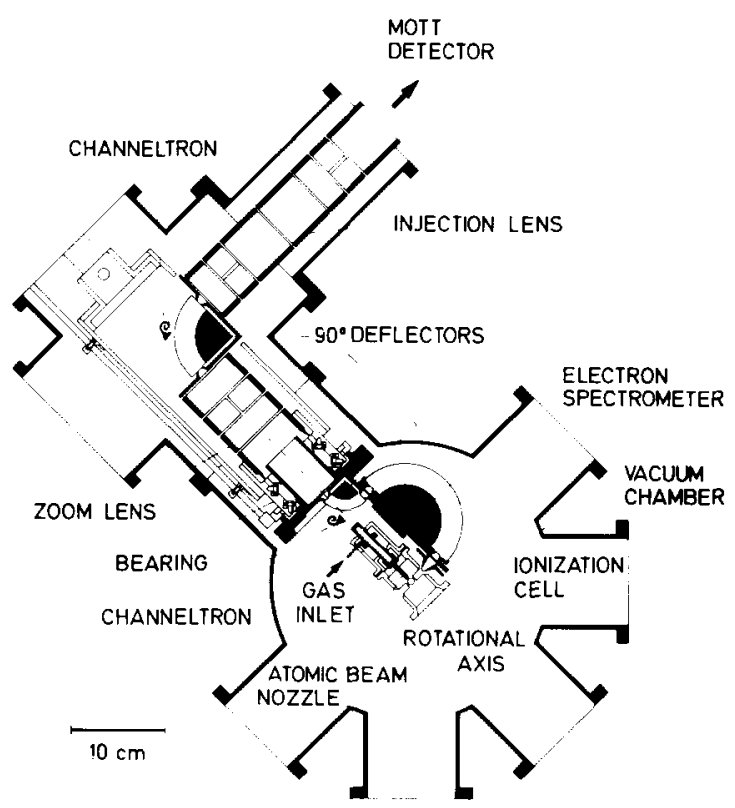

Fig. 2. Semitechnical drawing of the electron spectrometer system. 


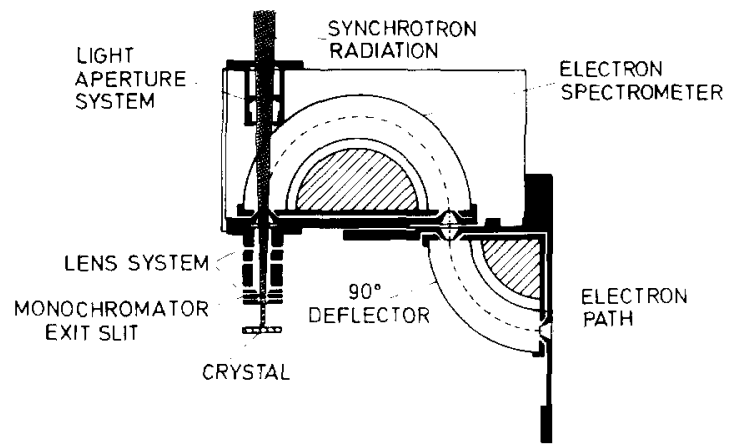

Fig. 3. Baffle system for measurements in normal incidence and normal emission.

where the electrons are decelerated and accelerated on their central path. The simulated hemispherical analyzer has the additional advantage that its geometric width is only about one-third that of a hemispherical spectrometer. As the spectrometer has to be rotated through the light beam, this property is important for the range of reaction angles $\theta$ to be scanned. A further advantage of the instrument is the possibility of using a compact fringe field correction [6], which allows a small distance between target point and entrance slit. The volume needed for a full rotation of the analyzer, where magnetic fields have to be effectively compensated, can therefore be kept smaller than with genuine hemispherical spectrometers. In addition, all electron-optical elements are machined from aluminum and coated with graphite, and $\mathrm{CuBe}$ ball bearings have been used. Presumably due to these precautions electrons with kinetic energies of $300 \mathrm{meV}$ could reliably be analyzed.

The construction of the $90^{\circ}$ deflectors is also based on the principle of the simulated spherical deflector. The lens systems employ standard cylindrical elements with deflector units. Two channeltrons behind the first and second deflector serve as monitors when the electron optics are adjusted.

The spectrometer system is operated with constant transmission energy and preacceleration. The ramp volt-

Table 1

Technical data of the electron spectrometer

\begin{tabular}{ll}
\hline $\begin{array}{l}\text { Central path diameter } \\
\text { Diameter of entrance and } \\
\text { exit slit }\end{array}$ & $100 \mathrm{~mm}$ \\
$\begin{array}{l}\text { Angular acceptance } \Delta \theta \\
\text { Angular range }\end{array}$ & $4 \mathrm{~mm}$ \\
$\quad$ (gas-phase version) & $\pm 5^{\circ}$ \\
Relative resolution $\Delta E / E$ & $+130^{\circ}$ to $-130^{\circ}$ \\
Typical resolution $\Delta E$ & $4.4 \%$ \\
& $240 \mathrm{meV}$ (at $5.5 \mathrm{eV}$ \\
& transmission energy) \\
& 90 meV (at $1.5 \mathrm{eV}$ \\
& transmission energy) \\
\hline
\end{tabular}

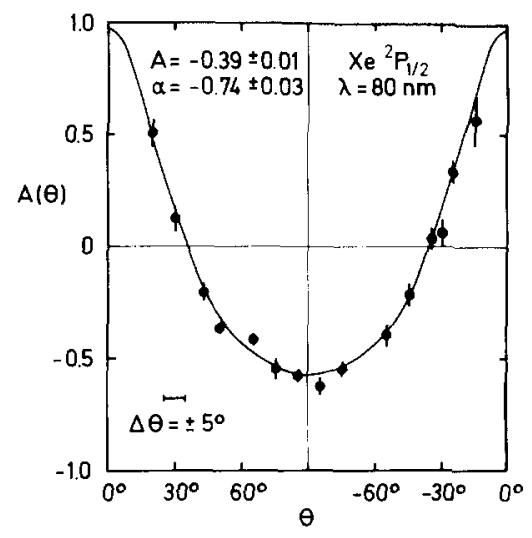

Fig. 4. Angular dependence of the spin polarization component $A(\theta)$ parallel to the photon momentum for completely circularly polarized light, for photoelectrons leaving the xenon ion in the ${ }^{2} \mathrm{P}_{1 / 2}$ state.

age needed is generated by an LSI 11 computer, which can be utilized for multichannel scaling. The LSI 11 also controls the data acquisition, including readout of counters, calculating spin polarization values, documentation of results on floppy disc and hard copy, and communicates with the microcomputer governing the monochromator control for setting the wavelength and choosing the light polarization.

Both systems have been in successful operation for more than two years [7-10]. The uhv version has seen more than a dozen bakeout cycles and the gas-phase version several hundred full rotations without any mechanical faults. After a painful period of adjustment, where the optimum potentials had to be found, the electron-optical parameters only have to be slightly re-optimized at the beginning of a new measuring period to account for changes of local parameters in the laboratory (e.g. stray magnetic fields), but are stable during one shift of typically two weeks.

As an example for the performance of the system. fig. 4 shows the angular dependence of the spin polarization component $A(\theta)$ for completely circularly polarized light for photoelectrons leaving the xenon ion in the $\mathrm{Xe}^{+2} \mathrm{P}_{1 / 2}$ state [7].

We thank the BESSY staff for fruitful cooperation and acknowledge support from the Bundesministerium für Forschung und Technologie.

\section{References}

[1] A. Eyers, Ch. Heckenkamp, F. Schäfers, G. Schönhense and U. Heinzmann, Nucl. Instr. and Meth. 208 (1983) 303.

[2] K.N. Huang, Phys. Rev. A22 (1980) 223.

[3] J. Kessler, Polarized Electrons, 2nd ed. (Springer, Berlin, 1985) p. $14 \mathrm{ff}$. 
[4] U. Heinzmann, J. Phys. B11 (1978) 399.

[5] K. Jost, J. Phys. E12 (1979) 1006.

[6] K. Jost, J. Phys. E12 (1979) 1001.

[7] Ch. Heckenkamp, F. Schäfers, G. Schönhense and U. Heinzmann, Phys. Rev. Lett. 52 (1984) 421.

[8] A. Eyers, F. Schäfers, G. Schönhense, U. Heinzmann,
H.P. Oepen, K. Hünlich, J. Kirschner and G. Borstel, Phys. Rev. Lett. 52 (1984) 1559.

[9] G. Schönhense, A. Eyers, U. Friess, F. Schäfers and U. Heinzmann, Phys. Rev. Lett. 54 (1985) 547.

[10] g. Schönhense, F. Schäfers, Ch. Heckenkamp. U. Heinzmann and M.A. Baig, J. Phys. B17 (1984) L771. 